\title{
Sensitivity of Schur stability of systems of linear difference equations with periodic coefficients
}

\author{
Ahmet Duman ${ }^{1}$, Gülnur Çelik Kızılkan ${ }^{1}$ and Kemal Aydın ${ }^{2}$ \\ ${ }^{1}$ Department of Mathematics and Computer Science, Necmettin Erbakan University, Konya, Turkey \\ ${ }^{2}$ Department of Mathematics, Selcuk University, Konya, Turkey
}

Received: 12 November 2015, Revised: 3 March 2016, Accepted: 4 March 2016

Published online: 18 April 2016.

\begin{abstract}
In this study, the sensitivity of Schur stability of systems of linear difference equations with periodic coefficients has been examined. The modified continuity theorems based on the parameters $\omega_{1}$ and $\omega_{2}$ have been given for Schur stability of linear difference equations with periodic coefficients. Also, new results have been obtained for sensitivity of $\omega^{*}-$ Schur stability based on the parameters $\omega_{1}$ and $\omega_{2}$. All the results have been applied to linear difference equations with periodic coefficients with order $k$. $k D-$ ball regions of Schur stability and $\omega^{*}$-Schur stability have been determined. In addition, the results related to $k D$-ball regions have been given.
\end{abstract}

Keywords: difference equation systems, monodromy matrix, periodic coefficients, Schur stability parameters, practical Schur stability, sensitivity.

\section{Introduction}

Let $A(n)$ is a $T$-periodic matrix with $N \times N$ dimension and $x(n)$ is an $N$ dimensional vector. Consider the following difference equation system:

$$
x(n+1)=A(n) x(n), x(0)=x_{0}, n \in \mathbb{Z} .
$$

Letting $T=1$, the coefficient $A(n)$ reduces to the constant coefficient $A(n+1)=A(n)=A$. In the present case, the Cauchy problem (1) becomes a Cauchy problem with constant coefficients as follows,

$$
x(n+1)=A x(n), x(0)=x_{0}, n \in \mathbb{Z} .
$$

The stability property of the Cauchy problem (2) with constant coefficients is well-known in the literature (see, for example, $[1,2,3])$. According to the spectral criterion, if all eigenvalues of the matrix $A$ belong to the unit disc, i.e. $\left|\lambda_{i}(A)\right|<1(i=1,2, \ldots, N)$, then the matrix $A$ is called to be an discrete-asymptotic stable matrix. Therefore, the system (2) is also called as discrete-asymptotic stable system (see, for example, [1,2,3,4]).

However, in the plane $C$, define the region $C_{S}=\{z \in C:|z|<1\}$. If $\sigma(A) \subset C_{S}$ then $A \in \mathbb{R}^{N \times N}$ is said to be Schur stable matrix, where $\sigma(A)$ is the spectrum of $A([5])$.

According to these statements, Schur stability and discrete-asymptotically stability are the equivalent concepts. Because of this equivalence, we will prefer to use the concept of Schur stability in this paper. 
It is well-known in the literature that eigenvalue problem of non-self-adjoint matrices is an ill-possed problem (see, for example, $[4,6])$. For this reason, the parameters revealing the quality of the stability obtained by avoiding calculation of eigenvalues are preferred to investigate stability.

Schur stability parameter $\omega(A)$ for the systems (2) is defined as:

$$
\omega(A)=\|H\| ; \quad H=\sum_{k=0}^{\infty}\left(A^{*}\right)^{k} A^{k}, \quad H=H^{*}>0, \quad A^{*} H A-H+I=0,
$$

where $I$ is unit matrix, $A^{*}$ is adjoint of the matrix $A,\|A\|=\max _{\|x\|=1}\|A x\|$ is the spectral norm of the matrix $A$, the norm $\|x\|$ is Euclidean norm for the vector $x=\left(x_{1}, x_{2}, \ldots, x_{N}\right)^{T}$. Linear difference system (2) is Schur stable if and only if $\omega(A)<\infty$ holds [7]. Let $1<\omega^{*} \in \mathbb{R}$ be the practical Schur stability parameter of the system (1). Then the matrix $A$ is called as practically Schur stable $\left(\omega^{*}-\right.$ Schur stable) if $\omega(A) \leq \omega^{*}$ holds. Otherwise the matrix $A$ is called as $\omega^{*}-$ Schur unstable matrix (see, for example, $[1,4,7]$ ). We should note here that the $\omega^{*}$ practical Schur stability parameter determined by the user according to their physical problem.

We shall be focused on the sensitivity of Schur stability of the linear difference equation system with periodic coefficients. The solution $X(n)$ of the equation

$$
X(n+1)=A(n) X(n), X(0)=I, n=0,1,2, \ldots
$$

is called a fundamental matrix of (1), where $A(n)=A(n+T)$ and the $X(T)$ is called a monodromy matrix defined as

$$
X(T)=\prod_{j=0}^{T-1} A(j)=A(T-1) A(T-2) \ldots A(1) A(0)
$$

(see, for example, $[1,2,8])$.

Similar to determinating the Schur stability of the coefficient matrix $A$ of the systems with constant coefficients given above, spectral criterion for Schur stability of monodromy matrix $X(T)$ of the system (1) is as follow.

If $\left|\lambda_{i}(X(T))\right|<1(i=1,2, \ldots, N)\left(\sigma(X(T)) \subset C_{S}\right)$ then the monodromy matrix $X(T)$ is Schur stable. Schur stability of the monodromy matrix $X(T)$ implies that the linear difference Cauchy problem with periodic coefficients (1) is Schur stable (see, for example, $[2,8]$ ). Schur stability parameters for the systems with periodic coefficients consist of two different parameters. First of the parameters is $\omega_{1}(A, T)$ which is given by

$$
\omega_{1}(A, T)=\|F\| ; F=\sum_{k=0}^{\infty}\left(X^{*}(T)\right)^{k}(X(T))^{k}, F=F^{*}>0 .
$$

If the Lyapunov difference matrix equation (LDME) $X^{*}(T) F X(T)-F+I=0$ has a positive defined symmetric solution $F=F^{*}>0$ then the linear difference system with periodic coefficients (1) is Schur stable $\left(\omega_{1}(A, T)<\infty\right)$, otherwise the system is not Schur stable.

Second of the parameters is $\omega_{2}(A, T)$ is given by

$$
\omega_{2}(A, T)=\|\Phi\| ; \Phi=\sum_{k=0}^{\infty} X^{*}(k) X(k), \Phi=\Phi^{*}>0 .
$$


Here $\Phi$ is given as follows,

$$
\Phi=\sum_{k=0}^{\infty}\left(X^{*}(T)\right)^{k} C(X(T))^{k}, C=\sum_{i=0}^{T-1} X^{*}(i) X(i), X^{*}(T) \Phi X(T)-\Phi+C=0
$$

The linear difference system with periodic coefficients (1) is Schur stable if and only if the $\operatorname{LDME} X^{*}(T) \Phi X(T)-\Phi+$ $C=0$ has a positive defined symmetric solution $\Phi=\Phi^{*}>0$ [8]. Furthermore, the system (1) is called as practically Schur stable by providing that $\omega_{i}(A, T) \leq \omega^{*}, i=1,2$. Otherwise, the system (1) is called as $\omega^{*}-$ Schur unstable [9].

\section{Sensitivity of Schur stability of systems of linear difference equations}

It is important to predict the behaviour of solutions of a problem and to know under which conditions similar properties are protected under perturbations to avoid the problem causes any chaos. The question "how much perturbation is ignorable for preserving the characteristic properties?" is known as the sensitivity problem.

In this section, we give some results in the literature on the sensitivity of the Schur stability of the systems with constant and periodic coefficients.

\subsection{Symbols}

Before introducing our theorems, we need to give the following definitions

$$
\begin{aligned}
& \alpha=\sum_{i=0}^{T-1}\|X(i)\|^{2}, \quad Q(n, s)=\prod_{j=s}^{n-1} A(j), \quad \Psi(n, s)=\prod_{j=s}^{n-1} B(j), \quad \gamma=(T-1) \max _{1 \leq k \leq T}\|Q(T, k)\|, \\
& \beta=\max _{1 \leq k \leq T}\|Q(T, k)\|\left(1+(T-1) \max _{1 \leq k \leq T-1}\|Q(k, 0)\|\right) \\
& \mu=\max _{1 \leq k \leq T}\|Q(T, k)\| \times\left\{\begin{array}{c}
\max _{0 \leq k \leq T-2}\|A(j)\| ; \quad \max _{0 \leq k \leq T-2}\|A(j)\| \leq 1 \\
\left(\max _{0 \leq k \leq T-2}\|A(j)\|\right)^{T-2} ; \max _{0 \leq k \leq T-2}\|A(j)\|>1
\end{array},\right. \\
& \Delta=Y(T)-X(T), \quad \Delta_{1}=\sqrt{\|X(T)\|^{2}+\frac{1}{\omega_{1}(A, T)}}-\|X(T)\|, \quad \Delta_{2}=\sqrt{\|X(T)\|^{2}+\frac{\alpha}{\omega_{2}(A, T)}}-\|X(T)\|, \\
& \Delta_{3}=\max _{0 \leq k \leq T-1}\|B(k)\|\left[\beta+\gamma \max _{1 \leq k \leq T-1}\|\Psi(T, k)\|+\mu \max _{1 \leq k \leq T}\|Q(T, k)\| \sum_{k=2}^{T-1 k-1} \sum_{l=1}\left(\frac{k !}{l !(k-l) !}\left(\max _{0 \leq j \leq k-1}\|B(j)\|\right)^{l}\right)\right], \\
& \Delta_{4}=\frac{\max _{1 \leq j, k \leq T}\|Q(j, k)\|\left(1+(T-1) \max _{1 \leq k \leq T-1}\|X(k)\|\right)}{1-(T-1) \max _{1 \leq j, k \leq T}\|Q(j, k)\| \max _{0 \leq k \leq T-1}\|B(k)\|} \max _{0 \leq k \leq T-1}\|B(k)\|, \quad \Delta_{5}=\sqrt{\|X(T)\|^{2}+\frac{1}{\omega_{2}(A, T)}}-\|X(T)\|, \\
& \Delta_{1}^{*}=\sqrt{\|X(T)\|^{2}+\frac{\omega^{*}-\omega_{1}(A, T)}{\omega^{*} \omega_{1}(A, T)}}-\|X(T)\|, \quad \Delta_{2}^{*}=\sqrt{\|X(T)\|^{2}+\frac{\omega^{*}-\omega_{2}(A, T)}{\omega^{*} \omega_{2}(A, T)}}-\|X(T)\| .
\end{aligned}
$$




\subsection{Sensitivity of Schur stability of systems with constant coefficients}

Let us give the following result which shows us how much perturbation is permissible for the autonomous difference equation system

$$
y(n+1)=(A+B) y(n), \quad n \in \mathbb{Z},
$$

where $B$ is a constant matrix with $N \times N$ dimensional. The system (3) is perturbated system of (2).

Theorem 1.Suppose that $A$ is a Schur stable matrix, that is $\omega(A)<\infty$. If the matrix $B$ satisfies $\|B\|<\sqrt{\|A\|^{2}+\frac{1}{\omega(A)}}-\|A\|$, then $A+B$ is Schur stable. Moreover, the inequality

$$
|\omega(A+B)-\omega(A)| \leq \frac{(2\|A\|+\|B\|)\|B\| \omega^{2}(A)}{1-(2\|A\|+\|B\|)\|B\| \omega(A)},
$$

holds ([10]; Theorem 4).

\subsection{Sensitivity of Schur stability of systems with periodic coefficients}

In the literature, some results are given under which conditions the perturbated system

$$
y(n+1)=(A(n)+B(n)) y(n), \quad B(n+T)=B(n), \quad n \in \mathbb{Z},
$$

preserves the Schur stability when the system (1) is Schur stable (see, for example, $[11,12])$. Some of these results give explicit conditions for bounds of the Schur stability parameters of the system (1) and its perturbated system (4), while some of the results provide bounds for the difference between the monodromy matrices $Y(T)$ and $X(T)$ of the systems (1) and (4), respectively. Such theorems in general known as the continuity theorems.

In this section, we give some continuity theorems of the system with periodic coefficients.

Theorem 2. Let the system (1) is Schur stable, $X(T)$ and $Y(T)$ be the monodromy matrices of (1) and (4), respectively.If the matrix $B(n)$ satisfies

$$
\|Y(T)-X(T)\|<\sqrt{\|X(T)\|^{2}+\frac{1}{\omega_{1}(A, T)}}-\|X(T)\|,
$$

then the system (4) is Schur stable ([11]; Theorem 2). Moreover, the inequality

$$
\|\widetilde{F}-F\| \leq \frac{\left(2\|X(T)\|\|Y(T)-X(T)\|+\|Y(T)-X(T)\|^{2}\right)\|F\|}{1-\left(2\|X(T)\|\|Y(T)-X(T)\|+\|Y(T)-X(T)\|^{2}\right)\|F\|} \omega_{1}(A, T)
$$

holds, where $\widetilde{F}=\sum_{k=0}^{\infty}\left(Y^{*}(T)\right)^{k}(Y(T))^{k}([11]$; Theorem 3).

Theorem 3. Let $X(T)$ and $Y(T)$ be the monodromy matrices of the systems (1) and (4), respectively, then $\|Y(T)-X(T)\| \leq \Delta_{3}$. Moreover, if (1) is Schur stable, then for the perturbation matrix $B(n)$ satisfying $\Delta_{3}<\Delta_{1}$, the system (4) is Schur stable too ([13]; Theorem 3).

Theorem 4. Let $X(T)$ and $Y(T)$ be the monodromy matrices of the systems (1) and (4), respectively, and $(T-1) \max _{1 \leq j, k \leq T}\|Q(j, k)\| \max _{0 \leq k \leq T-1}\|B(k)\|<1$, then we have $\|Y(T)-X(T)\| \leq \Delta_{4}$. Moreover, if (1) is Schur stable 
system, then (4) is Schur stable too provided that B(n) satisfies

$$
\|B(n)\|<\frac{\Delta_{1}}{\max _{1 \leq j, k \leq T}\|Q(j, k)\|\left[1+(T-1)\left(\max _{1 \leq k \leq T-1}\|X(k)\|+\Delta_{1}\right)\right]}
$$

([13]; Theorem 4).

Theorem 5. Let the system (1) be Schur stable, and $B(n)$ be a perturbation matrix satisfying each of the following conditions.

(i) $\Delta_{3}<\Delta_{2}$,

(ii) $\|B(n)\|<\frac{\Delta_{2}}{\max _{1 \leq j, k \leq T}\|Q(j, k)\|\left[1+(T-1)\left(\max _{1 \leq k \leq T-1}\|X(k)\|+\Delta_{2}\right)\right]}$,

then the perturbated system (4) is Schur stable too ([13]; Theorem 5).

\section{Main results}

\subsection{Sensitivity of linear difference equations systems with periodic coefficients}

In this part, we give upper bounds for difference between Schur stability parameters of the systems (1) and (4), upper bounds for Schur stability parameters of the system (4). In addition to the following lemma give a symmetric positive difinite matrix $C=C^{*}>0$ which satisfies LDME for Schur stable matrix $X(T)($ or $Y(T))$.

Lemma 1. Let the systems (1) and (4) be Schur stable.

(i) LDME of the system (1) is satisfied by symmetric positive matrix

$$
C=C_{2}+\Delta^{*} \widetilde{F} X(T)+X^{*}(T) \widetilde{F} \Delta+\Delta^{*} \widetilde{F} \Delta
$$

where the positive definite matrix $\widetilde{F}$ is solution of LDME of the system (4) for any a matrix $C_{2}=C_{2}^{*}>0$,

(ii) For any matrix $C_{1}=C_{1}^{*}>0$, in correspondence to the solution $F=F^{*}>0$ which satisfies LDME of system (1), the symmetric positive definite matrix satisfing LDME of the system (4) is

$$
C=C_{1}+\Delta^{*} F \Delta-\Delta^{*} F Y(T)-Y^{*}(T) F \Delta .
$$

Proof. (i) Since the system (1) is Schur stable, that is monodromy matrix $X(T)$ is Schur stable, there is a positive definite solution of LDME

$$
X^{*}(T) F X(T)-F+C_{1}=0 ; C_{1}=C_{1}^{*}>0
$$

which is $F=F^{*}>0$ and since the system (4) is also Schur stable, there is a positive definite solution of LDME

$$
Y^{*}(T) \widetilde{F} Y(T)-\widetilde{F}+C_{2}=0 ; C_{2}=C_{2}^{*}>0
$$

which is $\widetilde{F}=\widetilde{F}^{*}>0$. Lets seek the matrix $C$ satisfying

$$
X^{*}(T) \widetilde{F} X(T)-\widetilde{F}+C=0 ; C=C^{*}>0
$$


for $X(T)$ and $\widetilde{F}=\widetilde{F}^{*}>0$ which is the solution of (5). In equation (5), if we replace $Y(T)$ by $X(T)+\Delta$, it yields the following equation

$$
(X(T)+\Delta)^{*} \widetilde{F}(X(T)+\Delta)-\widetilde{F}=-C_{2}
$$

and we have

$$
X^{*}(T) \widetilde{F} X(T)-\widetilde{F}=-\left(C_{2}+\Delta^{*} \widetilde{F} X(T)+X^{*}(T) \widetilde{F} \Delta+\Delta^{*} \widetilde{F} \Delta\right)
$$

Thus we obtain LDME of the system (1) satisfied by symmetric positive matrix

$$
C=C_{2}+\Delta^{*} \widetilde{F} X(T)+X^{*}(T) \widetilde{F} \Delta+\Delta^{*} \widetilde{F} \Delta .
$$

(ii) The proof is similar to Lemma 1 (i) proof.

Example 1. Let $A(n)=\left(\begin{array}{cc}0.99 & 0 \\ 0 & \left(\frac{-1}{2}\right)^{n}\end{array}\right)$ for the system (1). Perturbate the system (1) with

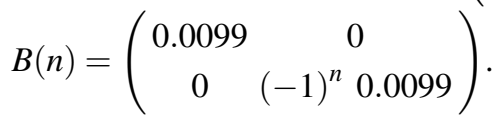

(i) According to Lemma 1 (i) $C=C^{*}=\left(\begin{array}{cc}197.03 & 0 \\ 0 & 1.26689\end{array}\right)$ is obtained which satisfies LDME of the system (1) for the symmetric positive definite matrix $C_{2}=\left(\begin{array}{cc}1.9998 & 0 \\ 0 & 1.26\end{array}\right)$ which satisfies LDME of the system (4),

(ii) According to Lemma 1 (ii), $C=C^{*}=\left(\begin{array}{cc}0.02009749 & 0 \\ 0 & 1.2432\end{array}\right)$ is obtained which satisfies LDME of the system (4) for the symmetric positive definite matrix $C_{1}=\left(\begin{array}{cc}1.9801 & 0 \\ 0 & 1.25\end{array}\right)$ which satisfies LDME of the system (1).

Now lets re-express Continuity Theorem given with Theorem 2 for the parameter $\omega_{1}(A, T)$ by considering Theorem 3 and 4.

Theorem 6. Let the system (1) be Schur stable, (i.e. $\left.\omega_{1}(A, T)<\infty\right)$. For the matrix $B(n)$ that satisfies the inequality

$$
\|B(n)\|<\frac{\Delta_{1}}{\max _{1 \leq j, k \leq T}\|Q(j, k)\|\left[1+(T-1)\left(\max _{1 \leq k \leq T-1}\|X(k)\|+\Delta_{1}\right)\right]}
$$

following inequalities hold

$$
\omega_{1}(A+B, T) \leq \frac{\omega_{1}(A, T)}{1-\left(2\|X(T)\|+\Delta_{4}\right) \Delta_{4} \omega_{1}(A, T)} ;\|\widetilde{F}-F\| \leq \frac{\left(2\|X(T)\|+\Delta_{4}\right) \Delta_{4} \omega_{1}(A, T)^{2}}{1-\left(2\|X(T)\|+\Delta_{4}\right) \Delta_{4} \omega_{1}(A, T)}
$$

Proof. The proof is similar to that of Theorem 2 and is obtained by replacing $\|Y(T)-X(T)\|$ by $\Delta_{4}$, so we omit the details.

Remark 1. In Theorem 2, the upper bounds in obtained inequalities depend on $\|Y(T)-X(T)\|$, thereby depends on the uncalculated matrix $Y(T)$ of perturbed system. On the other hand, in Theorem 6 , these upper bounds totally depend on the perturbation matrix $B(n)$ which guarantees the Schur stability of system (1). Therefore, the calculation of upper bounds in inequalities in Theorem 6 is more adventageous than that in Theorem 2. Furthermore, for perturbation matrix $B(n)$ that satisfies the boundary condition in the inequality $\|Y(T)-X(T)\|<\Delta_{1}$ in Theorem 2, if one uses $\Delta_{1}$ without calculating the matrix $Y(T)$ in inequalities in Theorem 6 , then the following equation is obtained:

$$
\left(2 \Delta_{1}\|X(T)\|+\Delta_{1}^{2}\right) \omega_{1}(A, T)=1 .
$$


In that case, inequalities in Theorem 2 would be meaningless.

Remark 2. For the perturbation matrix $B(n)$ which satisfies the condition $\Delta_{3}<\Delta_{1}$, the inequalities in Theorem 6 are also valid for $\Delta_{3}$.

Example 2. Let us consider the equation

$$
x(n+1)=\left(\begin{array}{cc}
0.99 & 0 \\
0 & \left(\frac{-1}{2}\right)^{n}
\end{array}\right) x(n) .
$$

The monodromy matrix of the system is

$$
X(2)=\left(\begin{array}{cc}
0.9801 & 0 \\
0 & -0.25
\end{array}\right)
$$

and its condition number is $\omega_{1}(A, 2)=25.3781$. Perturbation boundary whose Schur stability is guaranteed by Theorem 6 of given Schur stable system is $\|B(n)\|<0.000990101$. However, an obvious boundary like this cannot be given using Theorem 2. For the perturbation matrix

$$
B(n)=\left(\begin{array}{cc}
0.0099 & 0 \\
0 & (-1)^{n} 0.0099
\end{array}\right), \max _{0 \leq k \leq 1}\|B(k)\|=0.0099
$$

$\omega_{1}(A+B, 2)=2500.38$ and $\left|\omega_{1}(A+B, 2)-\omega_{1}(A, 2)\right|=2475.0019$. Using Theorem 6 without calculating matrix $Y(T)$ and only depending on the arguments of the system and matrix $B(n)$, right hand side of the first inequality is calculated as 244924.38 and right hand side of the second inequality is calculated as 244899.002378 . In contrast to that, to calculate the right hand sides of inequalities in Theorem 2, the matrix $Y(T)$ must be calculated first.

Expression of Theorem 6 for $T=1$.

Corollary 1. Suppose that the system (1)(the system (2)) is Schur stable. If the matrix B satisfies $\|B\|<\sqrt{\|A\|^{2}+\frac{1}{\omega(A)}}-$ $\|A\|$, then the system (4)(the system (3)) is Schur stable too. Moreover, the inequalities

$$
\omega(A+B) \leq \frac{\omega(A)}{1-(2\|A\|+\|B\|)\|B\| \omega(A)} ;\|\widetilde{F}-F\| \leq \frac{(2\|A\|+\|B\|)\|B\| \omega^{2}(A)}{1-(2\|A\|+\|B\|)\|B\| \omega(A)}
$$

hold.

Proof. For systems with periodic coefficients, in case of $T=1$, we have $A(n)=A, B(n)=B,\left.X(T)\right|_{T=1}=A$, $\left.\omega_{1}(A, T)\right|_{T=1}=\omega(A),\left.\Delta_{1}\right|_{T=1}=\sqrt{\|A\|^{2}+\frac{1}{\omega(A)}}-\|A\|$ and $\left.\Delta_{4}\right|_{T=1}=\|B\|$. Here, for convenience, we assume that $\max _{i \leq k \leq j, j<i}\{\}=$.0 and $\sum_{k=i, j<i}^{j}()=$.0 . Thus the proof is completed.

It is clear that, Corollary 1 is completely the same as Theorem 1 . This shows us that Theorem 6 is compatible with the results in literature. Now let us give a variant, in terms of the parameter $\omega_{2}$ of Theorem 6.

Theorem 7. Let the system (1) be Schur stable, ( i.e. $\left.\omega_{2}(A, T)<\infty\right)$. For a perturbation matrix $B(n)$ which satisfies the inequality

$$
\|B(n)\|<\frac{\Delta_{5}}{\max _{1 \leq j, k \leq T}\|Q(j, k)\|\left[1+(T-1)\left(\max _{1 \leq k \leq T-1}\|X(k)\|+\Delta_{5}\right)\right]}
$$


the system (4) is Schur stable. Moreover, the following inequalities hold

$$
\omega_{2}(A+B, T) \leq \frac{\omega_{2}(A, T)}{1-\left(2\|X(T)\|+\Delta_{4}\right) \Delta_{4} \omega_{2}(A, T)} ;\|\widetilde{F}-F\| \leq \frac{\left(2\|X(T)\|+\Delta_{4}\right) \Delta_{4} \omega_{2}(A, T)^{2}}{1-\left(2\|X(T)\|+\Delta_{4}\right) \Delta_{4} \omega_{2}(A, T)}
$$

Proof. The proof is easily obtained by considering the inequalities $\omega_{1}(A, T) \leq \omega_{2}(A, T), 1 / \omega_{1}(A, T) \leq \alpha / \omega_{2}(A, T)$ and using Theorem 6.

Remark 3. For perturbation matrix $B(n)$ which satisfies the condition $\Delta_{3}<\Delta_{5}$, inequalities given in Theorem 7 is also valid for $\Delta_{3}$. For $T=1$, the statement of Theorem 7 is the same as the statement of Corollary 1 . Indeed, in case of $T=1$, we have

$$
\begin{gathered}
A(n)=A, \quad B(n)=B,\left.\quad X(T)\right|_{T=1}=A,\left.\quad \omega_{2}(A, T)\right|_{T=1}=\omega(A), \\
\frac{\max _{1 \leq j, k \leq T}\|Q(j, k)\|\left[1+(T-1)\left(\max _{1 \leq k \leq T-1}\|X(k)\|+\Delta_{5}\right)\right]}{\Delta_{T=1}}=\sqrt{\|A\|^{2}+\frac{1}{\omega(A)}}-\|A\|, \\
\left.\frac{\omega_{2}(A, T)}{1-\left(2\|X(T)\|+\Delta_{4}\right) \Delta_{4} \omega_{2}(A, T)}\right|_{T=1}=\frac{\omega(A)}{1-(2\|A\|+\|B\|)\|B\| \omega(A)}, \\
\left.\frac{\left(2\|X(T)\|+\Delta_{4}\right) \Delta_{4} \omega_{2}(A, T)^{2}}{1-\left(2\|X(T)\|+\Delta_{4}\right) \Delta_{4} \omega_{2}(A, T)}\right|_{T=1}=\frac{(2\|A\|+\|B\|)\|B\| \omega^{2}(A)}{1-(2\|A\|+\|B\|)\|B\| \omega(A)} .
\end{gathered}
$$

Thus Corollary 1 is obtained.

Theorem 7, like Theorem 6, is also compatible with the results in literature.

Example 3. Let's consider the system $x(n+1)=A_{i}(n) x(n),(i=1,2)$ and

$$
A_{1}(n)=\left(\begin{array}{cc}
\frac{(-1)^{n}}{4} & 0.2 \\
0.4 & \frac{(-1)^{n}}{5}
\end{array}\right), A_{2}(n)=\left(\begin{array}{cc}
0.99 & \frac{3}{2} \\
0 & \frac{(-1)^{n}}{2}
\end{array}\right)
$$

For these systems we obtain $\omega_{1}\left(A_{1}, 2\right)=1.002, \omega_{1}\left(A_{2}, 2\right)=107.377$ and $\omega_{2}\left(A_{1}, 2\right)=1.30124, \omega_{2}\left(A_{2}, 2\right)=213.775$. These systems are Schur stable. Let's apply a perturbation to the system which has coefficient matrix $A_{1}(n)$ using following matrices

$$
B_{1}^{1}(n)=\left(\begin{array}{cc}
(-1)^{n} 0.2 & 0 \\
0 & 0.2
\end{array}\right), B_{1}^{2}(n)=\left(\begin{array}{cr}
(-1)^{n} 0.2 & 0 \\
0.1 & 0
\end{array}\right) .
$$

and apply another perturbaton to the system which has coefficient matrix $A_{2}(n)$ using following matrices

$$
B_{2}^{1}(n)=\left(\begin{array}{cc}
0.0001 & 0 \\
0 & (-1)^{n} 0.0001
\end{array}\right), B_{2}^{2}(n)=\left(\begin{array}{lc}
0 & 0.0001 \\
0 & (-1)^{n} 0.0001
\end{array}\right) .
$$

According to these matrices, following results are obtained.

$$
\begin{gathered}
\omega_{1}\left(A_{1}+B_{1}^{1}, 2\right)=1.05289, \omega_{1}\left(A_{1}+B_{1}^{2}, 2\right)=1.03229, \\
\omega_{1}\left(A_{2}+B_{2}^{1}, 2\right)=108.446, \omega_{1}\left(A_{2}+B_{2}^{2}, 2\right)=107.377, \\
\omega_{2}\left(A_{1}+B_{1}^{1}, 2\right)=1.5941, \omega_{2}\left(A_{1}+B_{1}^{2}, 2\right)=1.56593, \\
\omega_{2}\left(A_{2}+B_{2}^{1}, 2\right)=215.913, \omega_{2}\left(A_{2}+B_{2}^{2}, 2\right)=213.773 .
\end{gathered}
$$

Now, let us write some symbolic definitions for the difference between Schur stability parameters and the right hand sides of inequalities given in Theorem 6 and Theorem 7 for simplicity in notation. 
(1) $M=\left|\omega_{1}(A+B, T)-\omega_{1}(A, T)\right|$

(2) $N=\left|\omega_{2}(A+B, T)-\omega_{2}(A, T)\right|$

(3) $M_{i}=\frac{\left(2\|X(T)\|+\Delta_{i}\right) \Delta_{i} \omega_{1}(A, T)^{2}}{1-\left(2\|X(T)\|+\Delta_{i}\right) \Delta_{i} \omega_{1}(A, T)},(i=3,4)$

(4) $N_{i}=\frac{\left(2\|X(T)\|+\Delta_{i}\right) \Delta_{i} \omega_{2}(A, T)^{2}}{1-\left(2\|X(T)\|+\Delta_{i}\right) \Delta_{i} \omega_{2}(A, T)},(i=3,4)$

(5) $P_{i}=\frac{\omega_{1}(A, T)}{1-\left(2\|X(T)\|+\Delta_{i}\right) \Delta_{i} \omega_{1}(A, T)},(i=3,4)$

(6) $T_{i}=\frac{\omega_{2}(A, T)}{1-\left(2\|X(T)\|+\Delta_{i}\right) \Delta_{i} \omega_{2}(A, T)},(i=3,4)$.

Now considering the given data, let's comment on Theorems 6 and 7 using the Table 1 and Table 2.

\begin{tabular}{|l|l|l|l|l|l|l|l|}
\hline$A(n)$ & $B(n)$ & $M$ & $M_{3}$ & $M_{4}$ & $N$ & $N_{3}$ & $N_{4}$ \\
\hline \multirow{2}{*}{$A_{1}$} & $B_{1}^{1}$ & 0.05089 & 0.181966 & 0.226854 & 0.29286 & 0.324478 & 0.410326 \\
\cline { 2 - 8 } & $B_{1}^{2}$ & 0.03029 & 0.239043 & 0.314567 & 0.26469 & 0.434065 & 0.585393 \\
\hline \multirow{2}{*}{$A_{2}$} & $B_{2}^{1}$ & 1.069 & 41.0627 & 41.0711 & 2.138 & 262.058 & 262.145 \\
\cline { 2 - 8 } & $B_{2}^{2}$ & 0 & 41.3465 & 41.3551 & 0.002 & 264.987 & 265.076 \\
\hline
\end{tabular}

Table 1: This table illustrates the upper bounds of $M$ and $N$ in Theorem 6 and Theorem 7.

As seen in Table $1, M_{i}$ and $N_{i}(i=3,4)$ which are the upper bounds of differences of condition numbers of perturbed and non perturbed systems given in Theorem 6 and Theorem 7, are affected by perturbation and therefore when perturbation is changed, healthy information can be obtained about the change of difference between Schur stability parameters of system (1) and system (4). As seen in the table, the upper bounds $M_{i}$ and $N_{i}(i=3,4)$ of the differences $M$ and $N$ give closer results to occuring differences when the quality of Schur stability is stronger (i.e. the parameter value is smaller). For example, for the coefficient matrix $A_{1}(n)$ and the perturbation matrix $B_{1}^{2}(n)$, the occuring differences are $M=0.03029$ and $N=0.26469$ which has upper bounds $M_{3}=0.239043, M_{4}=0.314567$ and $N_{3}=0.434065, N_{4}=0.585393$ which are closer to occuring differences. However, for the coefficient matrix $A_{2}(n)$, which has lower quality of Schur stability with compare to $A_{1}(n)$, the upper bounds are much greater than differences occured.

\begin{tabular}{|l|l|l|l|l|l|l|l|}
\hline$A(n)$ & $B(n)$ & $\omega_{1}(A+B, T)$ & $P_{3}$ & $P_{4}$ & $\omega_{2}(A+B, T)$ & $T_{3}$ & $T_{4}$ \\
\hline \multirow{2}{*}{$A_{1}$} & $B_{1}^{1}$ & 1.05289 & 1.18397 & 1.22885 & 1.5941 & 1.62572 & 1.71157 \\
\cline { 2 - 8 } & $B_{1}^{2}$ & 1.03229 & 1.24104 & 1.31657 & 1.56593 & 1.73531 & 1.88663 \\
\hline \multirow{2}{*}{$A_{2}$} & $B_{2}^{1}$ & 108.446 & 148.44 & 148.448 & 215.913 & 475.833 & 475.92 \\
\cline { 2 - 8 } & $B_{2}^{2}$ & 107.377 & 148.723 & 148.732 & 213.773 & 478.762 & 478.851 \\
\hline
\end{tabular}

Table 2: This table illustrates the upper bounds of $\omega_{1}(A+B, T)$ and $\omega_{2}(A+B, T)$ in Theorem 6 and Theorem 7 .

As seen in Table 2, $P_{i}$ and $T_{i}(i=3,4)$ which are the upper bounds of parameters of Schur stability of the perturbation system given in Theorem 6 and Theorem 7, are affected by the perturbation, so when perturbation changes, healthy information can be obtained about Schur stability parameters of the system (4). As seen in the table, $P_{i}$ and $T_{i}(i=3,4)$, which are upper bounds of $\omega_{1}(A+B, T)$ and $\omega_{2}(A+B, T)$ give results which are quite closer to the value occured while the quality of Schur stability is strong. Indeed, for the coefficient matrix $A_{1}(n)$ and the perturbation matrix $B_{1}^{1}(n)$, the 
values are $\omega_{1}\left(A_{1}+B_{1}^{1}, 2\right)=1.05289$ and $\omega_{2}\left(A_{1}+B_{1}^{1}, 2\right)=1.5941$. The upper bounds $P_{3}=1.18397, P_{4}=1.22885$ and $T_{3}=1.62572, T_{4}=1.71157$ of these values are closer to these values. However, for the coefficient matrix $A_{2}(n)$ which has lower quality of Schur stability, the upper bounds are much greater than the values occured.

\section{$3.2 \omega^{*}-$ Schur stability of linear difference equation systems with periodic coefficients}

In this section, some results on the sensitivity of the $\omega^{*}-$ Schur stability are investigated.

Theorem 8. Let the system (1) be $\omega^{*}$-Schur stable ( i.e. $\omega_{i}(A, T) \leq \omega^{*}, i=1,2$ ), and $B(n)$ be a perturbation matrix satisfying each of the following conditions:

(i) $\Delta_{3} \leq \Delta_{i}^{*} ; i=1,2$,

(ii) $\|B(n)\| \leq \frac{\Delta_{i}^{*}}{\max _{1 \leq j, k \leq T}\|Q(j, k)\|\left[1+(T-1)\left(\max _{1 \leq k \leq T-1}\|X(k)\|+\Delta_{i}^{*}\right)\right]} ; i=1,2$,

then the perturbated system (4) is $\omega^{*}-$ Schur stable too.

Proof. (i) Since $\frac{\omega^{*}-\omega_{i}(A, T)}{\omega^{*} \omega_{i}(A, T)}<\frac{1}{\omega_{i}(A, T)} \leq \frac{\alpha}{\omega_{i}(A, T)}$, the inequality $\Delta_{3} \leq \Delta_{i}^{*}<\Delta_{i}(i=1,2)$ is hold. Thus the perturbated system (4) is Schur stable according to Theorem 3 and 5 . Therefore if the inequality

$$
\Delta_{3} \leq \Delta_{i}^{*}=\sqrt{\|X(T)\|^{2}+\frac{\omega^{*}-\omega_{i}(A, T)}{\omega^{*} \omega_{i}(A, T)}}-\|X(T)\|, i=1,2
$$

is solved for $\omega^{*}$, the inequality

$$
\frac{\omega_{i}(A, T)}{1-\left(2\|X(T)\|+\Delta_{3}\right) \Delta_{3} \omega_{i}(A, T)} \leq \omega^{*}, i=1,2
$$

is obtained. Thus the system (4) is $\omega^{*}-$ Schur stable from Theorem 6 and 7 and Remark 1 . and 2.

(ii) If the inequality $\|B(n)\| \leq \frac{\Delta_{i}^{*}}{\max _{1 \leq j, k \leq T}\|Q(j, k)\|\left[1+(T-1)\left(\max _{1 \leq k \leq T-1}\|X(k)\|+\Delta_{i}^{*}\right)\right]}$ is solved for $\Delta_{i}^{*}, i=1,2$, the inequality

$$
\Delta_{4} \leq \Delta_{i}^{*}, i=1,2
$$

is obtained. Since $\frac{\omega^{*}-\omega_{i}(A, T)}{\omega^{*} \omega_{i}(A, T)}<\frac{1}{\omega_{i}(A, T)} \leq \frac{\alpha}{\omega_{i}(A, T)}$, the inequality $\Delta_{4} \leq \Delta_{i}^{*}<\Delta_{i}(i=1,2)$ is hold. Thus the perturbated system (4) is Schur stable. Therefore if the inequality

$$
\Delta_{4} \leq \Delta_{i}^{*}=\sqrt{\|X(T)\|^{2}+\frac{\omega^{*}-\omega_{i}(A, T)}{\omega^{*} \omega_{i}(A, T)}}-\|X(T)\|, i=1,2
$$

is solved for $\omega^{*}$, the inequality

$$
\frac{\omega_{i}(A, T)}{1-\left(2\|X(T)\|+\Delta_{4}\right) \Delta_{4} \omega_{i}(A, T)} \leq \omega^{*}, i=1,2
$$

is obtained. Thus the system (4) is $\omega^{*}-$ Schur stable from Theorem 6 and 7.

Example 4. For the system (1) let $A(n)=\left(\begin{array}{cc}0.9 & 0.1 \\ 0 & \frac{(-1)^{n}}{10}\end{array}\right)$ and $\omega^{*}=10$. Since $\omega_{1}(A, 2)=2.93644 \leq \omega^{*}$ and $\omega_{2}(A, 2)=$ $5.32484 \leq \omega^{*}$, the system (1) is $\omega^{*}-$ Schur stable. For perturbation matrix $B(n)$ satisfying the inequalities $\|B(n)\| \leq$ 
0.0647958, $\|B(n)\| \leq 0.0269713$ and $\|B(n)\| \leq 0.0666285,\|B(n)\| \leq 0.0266247$ which are obtained using Theorem 8 (i) and Theorem 8 (ii), respectively, the system (4) is $\omega^{*}-$ Schur stable. For example, let the perturbation matrix be $B(n)=\left(\begin{array}{cc}0.026 & 0 \\ 0 & (-1)^{n} 0.026\end{array}\right)$, then $\|B(n)\|=0.026$. Indeed for the system $(4), A(n)+B(n)=\left(\begin{array}{cc}0.926 & 0.1 \\ 0 & (-1)^{n} 0.126\end{array}\right)$ and it can be seen that $\omega_{1}(A+B, 2)=3.81806 \leq \omega^{*}$ and $\omega_{2}(A+B, 2)=7.10177 \leq \omega^{*}$.

\subsection{Application of the results on the sensitivity of linear difference equations with order $k$}

Consider the following linear difference equations with order $k$

$$
x(n+1)-a_{0}(n) x(n)-\ldots-a_{k-1}(n) x(n-k+1)=0
$$

for $n \geq 0$ and $a_{i}(n)=a_{i}(n+T), i=0,1,2, \ldots, k-1, T>0$. By taking $x(n-k+1)=y_{1}(n), x(n-k+2)=y_{2}(n), \ldots$, $x(n)=y_{k}(n)$ the equation (6) can be written as

$$
y(n+1)=C(n) y(n), n \geq 0 \text { and } C(n+T)=C(n)
$$

in matrix-vector form, where the matrix $C(n)$ is companion matrix as follows

$$
C(n)=\left(\begin{array}{ccccc}
0 & 1 & 0 & \cdots & 0 \\
0 & 0 & 1 & \cdots & 0 \\
\vdots & \vdots & \vdots & \ddots & \vdots \\
0 & 0 & 0 & \cdots & 1 \\
a_{k-1}(n) & a_{k-2}(n) & a_{k-3}(n) & \cdots & a_{0}(n)
\end{array}\right) .
$$

Thus, the results on the sensitivity of Schur stability which are given for the system (1) can easily be used for the sensitivity of Schur stability of the linear difference equations with order $k(6)$.

Consider the perturbation of the equation (6), and so, of the system (7)

$$
z(n+1)=(C(n)+D(n)) z(n), n \geq 0, D(n+T)=D(n),
$$

where

$$
D(n)=\left(\begin{array}{ccccc}
0 & 0 & 0 & \cdots & 0 \\
0 & 0 & 0 & \cdots & 0 \\
\vdots & \vdots & \vdots & \ddots & \vdots \\
0 & 0 & 0 & \cdots & 0 \\
d_{k-1}(n) & d_{k-2}(n) & d_{k-3}(n) & \cdots & d_{0}(n)
\end{array}\right),
$$

$d_{i}(n)=d_{i}(n+T), T \geq 0, i=0,1,2, \ldots, k-1$.

The set $B_{\delta}$ called as the $k D$-ball, i.e. the $k$-dimensional ball [14], and defined as $B_{\delta}=\left\{x=\left(x_{1}, x_{2}, \ldots, x_{k}\right) \mid\|x(n)\|<\delta\right\}$. Let

- $d(n)=\left(d_{k-1}(n), d_{k-2}(n), \ldots, d_{1}(n), d_{0}(n)\right)$,

- $\delta_{i}=\frac{\Delta_{i}}{\max _{1 \leq j, k \leq T}\|Q(j, k)\|\left[1+(T-1)\left(\max _{1 \leq k \leq T-1}\|X(k)\|+\Delta_{i}\right)\right]}, i=1,2$, 


$$
\text { - } \delta_{i}^{*}=\frac{\Delta_{i}^{*}}{\max _{1 \leq j, k \leq T}\|Q(j, k)\|\left[1+(T-1)\left(\max _{1 \leq k \leq T-1}\|X(k)\|+\Delta_{i}^{*}\right)\right]}, i=1,2 .
$$

For the system (7), the variant of Theorem 4 is as follows.

Theorem 9. Let the system (7) be Schur stable. If the $k$-tuple $d(n) \in B_{\delta_{i}}(i=1,2)$, then the perturbed system (8) is Schur stable.

Proof. Since $d(n) \in B_{\delta_{i}}$, we obtain $\|D(n)\|=\|d(n)\|<\delta_{i}$. Therefore, if the system (7) is Schur stable, the condition $\|D(n)\|<\delta_{i} ; i=1,2$ in Theorems 4 and 5 guarantees the Schur stability of the perturbed system (8).

Remark 4. The $k D-$ ball $B_{\delta}$ occurs as a region of Schur stability for the perturbation matrix $D(n)$. The $1 D-$ ball $B_{\delta}$ is an interval, the $2 D$-ball $B_{\delta}$ is a disc, and the $3 D$-ball $B_{\delta}$ is the interior of a sphere, i.e. a solid ball.

Theorem 10. Let the system (7) be $\omega^{*}-$ Schur stable. If the $k$-tuple $d(n) \in B_{\delta_{i}^{*}}(i=1,2)$, then the perturbed system (8) is also $\omega^{*}-$ Schur stable.

Proof. The proof is easily obtained from Theorems 8 (ii) and 9.

Example 5. Consider the delay difference equation

$$
x(n+1)-\frac{1}{4} \cos (n \pi) x(n)=-\frac{1}{100} x(n-1), n \geq 0 .
$$

For the companion matrix $C(n)$, it is easy to check that $\omega_{1}(C, 2)=1.06833$ and $\omega_{2}(C, 2)=2.13647$. Therefore, the equation (9) is Schur stable.

- $\delta_{1}=0.259469, \delta_{2}=0.263176$,

- for $\omega_{1}^{*}=3 ; \delta_{1_{(3)}}^{*}=0.209221$ and $\delta_{2_{(3)}}^{*}=0.0828397$,

- for $\omega_{2}^{*}=10 ; \delta_{1_{(10)}^{*}}^{*}=0.246093$ and $\delta_{2_{(10)}^{*}}^{*}=0.159556$.

Consider the perturbed equation

$$
y(n+1)-\left(\frac{1}{4} \cos (n \pi)+d_{0}(n)\right) y(n)=\left(-\frac{1}{100}+d_{1}(n)\right) y(n-1),
$$

where $n \geq 0$ and $d_{i}(n)=d_{i}(n+2), i=0,1$.

- The equation (10) for all elements of the set $B_{\delta_{i}}(i=1,2)$ is Schur stable,

- The equation (10) for all elements of the set $B_{\delta_{i(3)}^{*}}(i=1,2)$ is 3-Schur stable,

- The equation (10) for all elements of the set $B_{\delta_{i(10)}^{*}}^{*}(i=1,2)$ is $10-$ Schur stable.

For $d(n)=\left((-1)^{n+1} 0.183,(-1)^{n} 0.183\right)$, perturbate matrix

$$
D(n)=\left(\begin{array}{cc}
0 & 0 \\
(-1)^{n+1} 0.183 & (-1)^{n} 0.183
\end{array}\right), \quad \max _{0 \leq k \leq 1}\|D(k)\|=0.258801
$$

While the system is perturbated by $D(n)$ we have $\omega_{1}(C+D, 2)=1.24149$ and $\omega_{2}(C+D, 2)=2.09479$ hence it is Schur stable. 


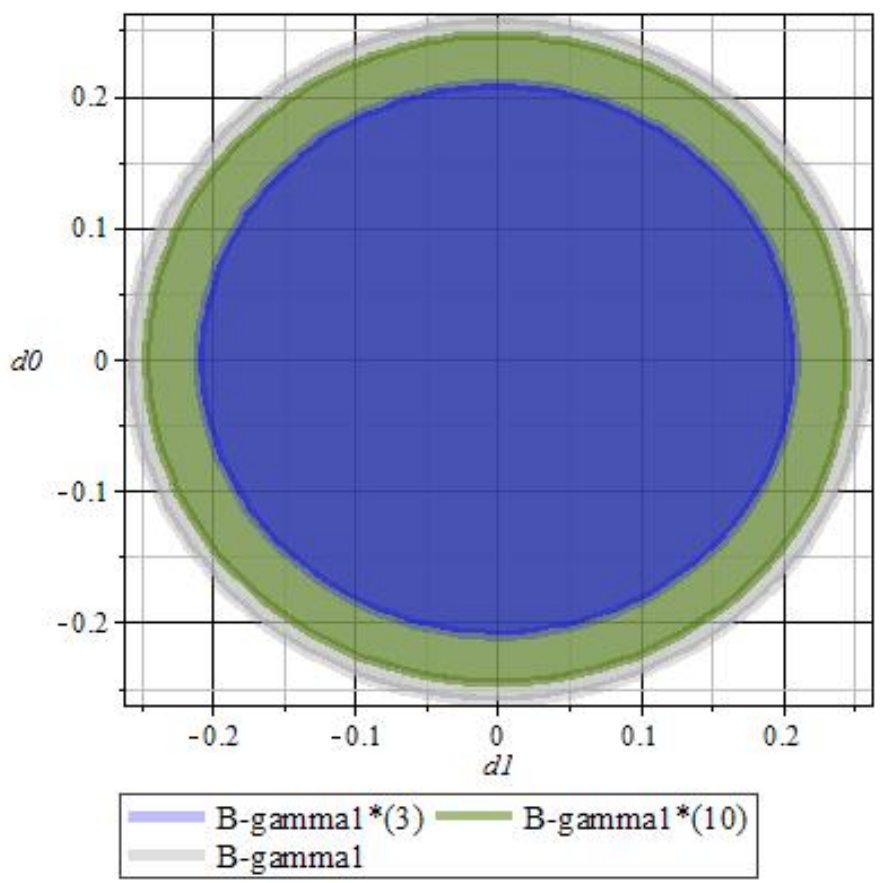

Fig. 1: Schur stability region of $B_{\gamma_{1}^{*}}, B_{\gamma_{1}^{*}}$ and $B_{\gamma_{1}}$.

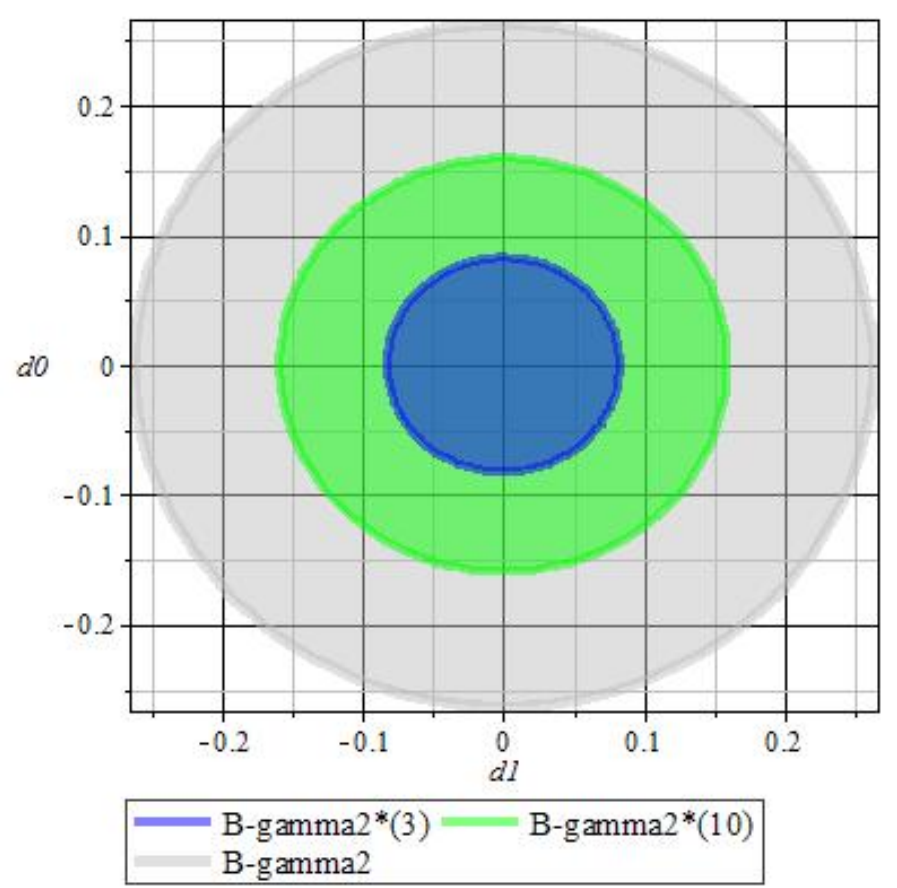

Fig. 2: Schur stability region of $B_{\gamma_{2}^{*}(3)}, B_{\gamma_{2}^{*}(10)}$ and $B_{\gamma_{2}}$. 
Schur stability region $B_{\delta_{i}}, 3$-Schur stability region $B_{\delta_{i_{(3)}}^{*}}$ and 10-Schur stability region $B_{\delta_{i_{(10)}}^{*}}$ of the equation (10) have been given with Figure 1 and Figure 2. As it is clearly seen from Figure 1 and Figure 2, $B_{\delta_{i_{(3)}}^{*}} \subset B_{\delta_{i_{(10)}}^{*}} \subset B_{\delta_{i}}(i=1,2)$.

Theorem 11. (i) $\lim _{\omega_{i} \rightarrow \infty} B_{\delta_{i}}=\varnothing, i=1,2$,

(ii) $\lim _{\omega_{i} \rightarrow \omega_{i}^{*}} B_{\delta_{i}^{*}}=\varnothing, i=1,2$,

(iii) $\lim _{\omega_{i}^{*} \rightarrow \infty} B_{\delta_{i}^{*}}=B_{\delta_{i}}, i=1,2$.

Proof. (i) The equality $\lim _{\omega_{i} \rightarrow \infty} \delta_{i}=0$ holds, where $\lim _{\omega_{i} \rightarrow \infty} \Delta_{i}=0, \quad i=1,2$. Therefore, $\lim _{\omega_{i} \rightarrow \infty}\left\{x=\left(x_{1}, x_{2}, \ldots, x_{n}\right) \mid\|x(n)\|<\delta_{i}\right\}=\varnothing$,

(ii) $\lim _{\omega_{i} \rightarrow \omega_{i}^{*}} B_{\delta_{i}^{*}}=\varnothing$ for $\lim _{\omega_{i} \rightarrow \omega_{i}^{*}} \delta_{i}^{*}=0, i=1,2$,

(iii) $\lim _{\omega_{i} \rightarrow \omega_{i}^{*}} B_{\delta_{i}^{*}}=\varnothing$ for $\lim _{\omega_{i} \rightarrow \omega_{i}^{*}} \delta_{i}^{*}=0, i=1,2$, so the proof is obtained.

Theorem 12. (i) The sequence of set $\left\{B_{\delta_{i}}\right\}$ is increasing according to $\delta_{i}, i=1,2$,

(ii) The sequence of set $\left\{B_{\delta_{i}^{*}}\right\}, i=1,2$ is bounded.

Proof. (i) Let $x(n+1)=C_{1}(n) x(n)$ and $y(n+1)=C_{2}(n) y(n)$. It is clear that if $\delta_{i}\left(C_{2}\right)<\delta_{i}\left(C_{1}\right)$ then the inclusion $B_{\delta_{i}\left(C_{2}\right)} \subset B_{\delta_{i}\left(C_{1}\right)}$ holds, $i=1,2$,

(ii) $\varnothing \subset B_{\delta_{i}^{*}} \subset B_{\delta_{i}}$ for $0<\delta_{i}^{*}<\frac{\Delta_{i}}{\max _{1 \leq j, k \leq T}\|Q(j, k)\|\left[1+(T-1)\left(\max _{1 \leq k \leq T-1}\|X(k)\|+\Delta_{i}\right)\right]}, i=1,2$.

Remark 5. The numerical examples have been computed by using matrix vector calculator MVC [15].

\section{Conclusion}

In this paper we have consider sensitivity problem for Schur stable linear difference equation system with periodic coefficients. For this problem, the upper bounds in obtained inequalities which depends on $\|Y(T)-X(T)\|$ in continuity theorems in literature have depend on the uncalculated matrix $Y(T)$ of perturbed system. On the other hand, the similar bounds in the continuity theorems in this study totally have depend on the perturbation matrix $B(n)$ which guarantees the Schur stability of system (1). Therefore, the calculation of upper bounds in inequalities in the continuity theorems have been more adventageous than that in continuity theorems in literature.

In addition, some new results on the sensitivity of $\omega^{*}-$ Schur stability have obtained. All the results have applied to linear difference equations with periodic coefficients with order $k$. $k D$-ball regions of Schur stability and $\omega^{*}-$ Schur stability have determined. Also some examples illustrating the efficiency of the theorems have given.

\section{References}

[1] Akın Ö. and Bulgak H., Linear difference equations and stability theory, Konya; Selçuk University, Research Center of Applied Mathematics, (1998). (in Turkish) 
[2] Elaydi S.N., An introduction to difference equations, New York; Springer-Verlag, (1999).

[3] Godunov S.K., Modern aspects of linear algebra, RI: American Mathematical Society, Translation of Mathematical Monographs 175. Providence, (1998).

[4] Bulgak H., "Pseudoeigenvalues, spectral portrait of a matrix and their connections with different criteria of stability", Error Control and Adaptivity in Scientific Computing, NATO Science Series, Series C: Mathematical and Physical Sciences, in: H. Bulgak and C. Zenger(Eds), Kluwer Academic Publishers, (1999), 536, 95-124.

[5] Voicu M. and Pastravanu O., "Generalized matrix diagonal stability and linear dynamical systems", Linear Algebra and its Applications, (2006), 419, 299-310.

[6] Wilkinson J. H., "The algebraic eigenvalue problem”, Clarendom Pres Oxford, (1965).

[7] A.Ya. Bulgakov (H. Bulgak), "An effectively calculable parameter for the stability quality of systems of linear differential equations with constant coefficients", Siberian Math. J. (1980), 21, 339-347.

[8] Aydın K., Bulgak H. and Demidenko G. V., "Numeric characteristics for asymptotic stability of solutions to linear difference equations with periodic coefficients", Siberian Mathematical Journal, (2000), 41, 1005-1014.

[9] Aydın K., "Schur stable difference equations with constant coefficients", Zonguldak Karaelmas University, Integral Geometry and Inverse Problem Workshop, May 08-09,(2004), Zonguldak.

[10] Duman A. and Aydın K., "Sensitivitiy of linear difference equation systems with constant coefficients", Scientific Research and Essays, (2011), 6(28) 5846-5854.

[11] Aydın K., Bulgak H. and Demidenko G. V., "Continuity of numeric characteristics for asymptotic stability of solutions to linear difference equations with periodic coefficients", Selçuk Journal Applied Mathematics, (2001), 2, 5-10.

[12] Aydın K., Bulgak H. and Demidenko G. V., "Asymptotic stability of solutions to perturbed linear difference equations with periodic coefficients", Siberian Mathematical Journal, (2002), 43, 389-401.

[13] Duman A. and Aydın K., "Sensitivity of Schur stability of monodromy matrix", Applied Mathematics and Computation, (2011), 217, 6663-6670.

[14] Roger AH., Charles RJ., Matrix analysis, Cambridge University Press, Cambridge, (1999).

[15] Bulgak H. and Eminov D., “Computer dialogue system MVC”, Selçuk Journal Applied Mathematics, (2001), 2, 17-38. 